Afrika Statistika

Afrika Statistika

Vol. 10(2), 2015, pages 815-826.

DOI: http://dx.doi.org/10.16929/as/2015.815.72

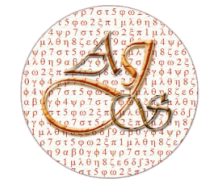

ISSN 2316-090X

\title{
Some inferences on truncated generalized Cauchy distribution
}

\author{
Mohammad Ahsanullah \\ Department of Management Sciences, Rider University Lawrenceville, New Jersey, 08648, USA
}

Received May 10, 2015; Accepted October 15, 2015

Copyright (C) 2015, Afrika Statistika. All rights reserved

\begin{abstract}
Rider (1957) introduced the generalized Cauchy distribution. In this paper we will consider a truncated version of the generalized Cauchy distribution. One possible use of the model is in life testing problem when the domain of application is non negative. Here we will consider several distributional properties of the truncated generalized Cauchy distribution. Some characterizations of this distribution based on truncated moments, order statistics and record values, will be given.

Résumé. Rider a introduit la distribution généralisée de Cauchy en 1957. Dans ce papier, nous étudions une version tronquée de cette distribution. Une utilisation potentielle de cette distribution concerne le test de survie lorsque le domaine d'application est positif. Nous allons démontrer plusieurs propriété de cette distribution. Nous donnerons aussi quelques de ses caractérisations, basées sur les moments tronqués, sur les statistiques d'ordre et sur les valeurs des records.
\end{abstract}

Key words: Order statistics; Record values; Characterization; Hazard rate.

AMS 2010 Mathematics Subject Classification : Primary : 62E15, 62E10. Secondary: $62 \mathrm{G} 30$

\section{Introduction}

The Cauchy distribution named after Agustin Cauchy is a continuous distribution. It is also known among physicists as Lorentz distribution (after Hendrik Lorentz). The probability density function(pdf) $f_{c}(x)$ of standard Cauchy with location parameter as zero and scale parameter 1 is as follows:

$$
f_{c}(x)=\frac{1}{\pi} \frac{1}{1+x^{2}}, \quad-\infty<x<\infty .
$$

Email: ahsan@rider.edu 
The mean of the standard Cauchy distribution does not exist. Rider (1957) introduced the generalized Cauchy distribution with $p d f f_{g c}(\mathrm{n} . \mathrm{x})$ as given below.

$$
f_{g c}(n, x)=\frac{\Gamma(n)}{\Gamma\left(\frac{1}{2}\right) \Gamma\left(n-\frac{1}{2}\right)} \frac{1}{\left(1+x^{2}\right)^{n}},-\infty<x<\infty, \quad n>1
$$

The $k t h$-moments of this distribution is finite if $k<2 n-1$. We will consider here the left truncated generalized Cauchy distribution truncated at zero. The pdf of the truncated generalized Cauchy distribution $f_{t g c}(n, x)$ is as follows:

$$
f_{t r c}(x)=\frac{2 \Gamma(n)}{\Gamma\left(\frac{1}{2}\right) \Gamma\left(n-\frac{1}{2}\right)} \frac{1}{\left(1+x^{2}\right)^{n}}, 0<x<\infty, \quad n>1 .
$$

We will call the random variable $X$ has the distribution $T G C(n, x)$ if its pdf is as given in (3). Ateya and Al-Hussaini (2011) gave various estimations of the location and scale parameters of this distribution.

In this paper we will consider several basic properties of this distribution. Based on these distributional properties, some characterizations of this distribution will be given.

\section{Main Results}

The following graphs in Figure 1 give the $p d f s$ of $T G C(n, x)$ for $n=2$ (red), $n=5$ (Black) and $n=10$ (Green).

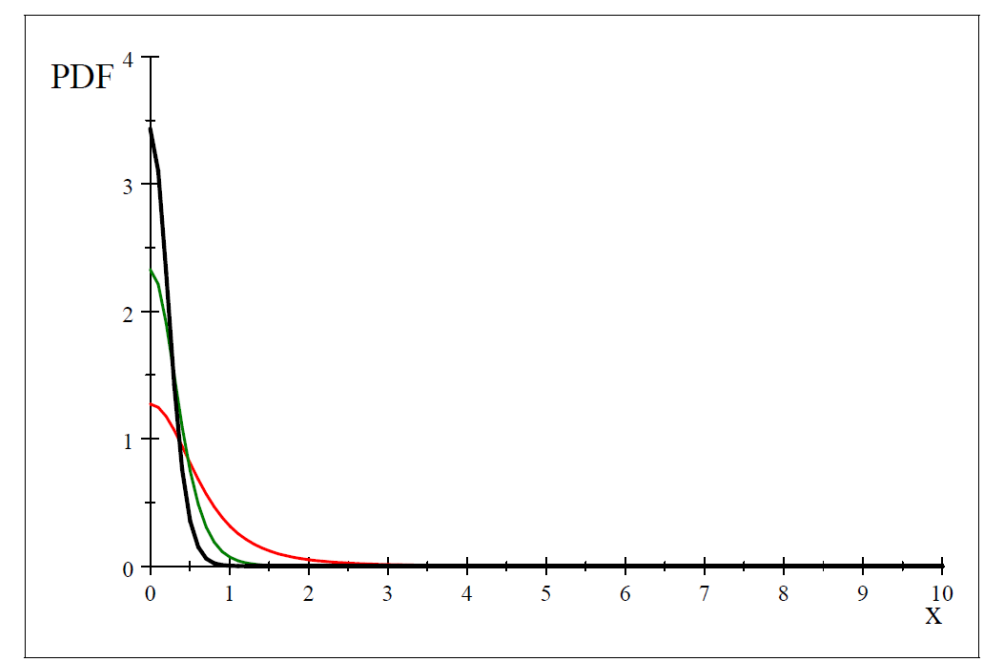

Fig. 1. PDFs

Journal home page: www.jafristat.net 
The cumulative distribution of $T G C(n, x)$ is as follows

$$
\begin{aligned}
F_{T G C}(x) & =\int_{0}^{x} \frac{2 \Gamma(n)}{\Gamma\left(\frac{1}{2}\right) \Gamma\left(n-\frac{1}{2}\right)} \frac{1}{\left(1+u^{2}\right)^{n}} d u \\
& =\frac{\Gamma(n)}{\Gamma\left(\frac{1}{2}\right) \Gamma\left(n-\frac{1}{2}\right)} \int_{\frac{1}{r^{1+x_{2} u}}}^{1} t^{n-\frac{1}{2}-1}(1-t)^{-\frac{1}{2}} d t \\
& =1-I_{\frac{1}{1+x^{2}}}\left(n-\frac{1}{2}, \frac{1}{2}\right),
\end{aligned}
$$

where

$$
I_{\frac{1}{1+x^{2}}}\left(n-\frac{1}{2}, \frac{1}{2}\right)=\frac{\Gamma(n)}{\Gamma\left(\frac{1}{2}\right) \Gamma\left(n-\frac{1}{2}\right)} \int_{0}^{\frac{1}{1+x_{2} \lll}} t^{n-\frac{1}{2}-1}(1-t)^{-\frac{1}{2}} d t
$$

The $c d f s$ of $T G C$ are given in Figure 2. CDF : Red for $n=2$, Green for $n=5$ and Black for $n=10$.

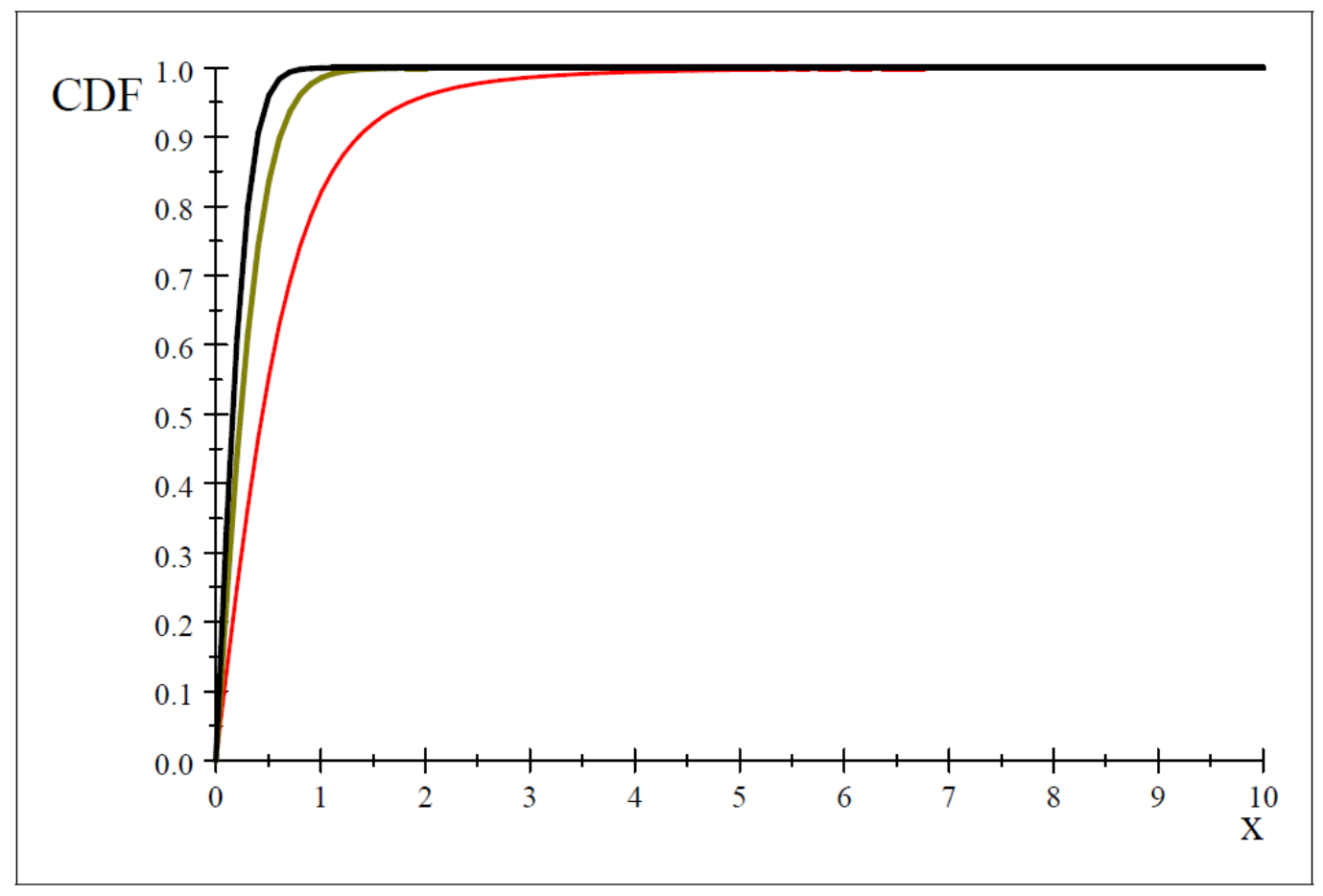

Fig. 2. CDFs of TGC 
Let $M_{T G C}(n)$ be the moment generating function of $T G C(n)$, then

$$
\begin{aligned}
M_{T G C}(n) & =\int_{0}^{\infty} \frac{2 \Gamma(n)}{\Gamma\left(\frac{1}{2}\right) \Gamma\left(n-\frac{1}{2}\right)} \frac{e^{t x}}{\left(1+x^{2}\right)^{n}} d x \\
& =\sum_{j=0}^{2 n-2} \frac{t^{j}}{i !} \frac{\Gamma(n)}{\Gamma\left(\frac{1}{2}\right) \Gamma\left(n-\frac{1}{2}\right)} B\left(n-\frac{j+1}{2}, \frac{j+1}{2}\right) \\
& =\sum_{j=0}^{2 n-2} \frac{t^{j}}{i !} \frac{\Gamma\left(n-\frac{j+1}{2}\right) \Gamma\left(\frac{j+1}{2}\right)}{\Gamma\left(\frac{1}{2}\right) \Gamma\left(n-\frac{1}{2}\right)} .
\end{aligned}
$$

The $k t h$ moment about zero $\mu_{k}(n)$ is given by

$$
\mu_{k}(n)=\frac{\Gamma\left(n-\frac{k+1}{2}\right) \Gamma\left(\frac{k+1}{2}\right)}{\sqrt{\pi} \Gamma\left(n-\frac{1}{2}\right)}, k<2 n-1 .
$$

The mean $\mu_{1}(n)$ of $T G C(n, x)$ is

$$
\begin{aligned}
\mu_{1}(n) & =E(X)=\frac{\Gamma(n-1)}{\Gamma\left(\frac{1}{2}\right) \Gamma\left(n=\frac{1}{2}\right)}, n>1 . \\
& =\frac{(n-2) !}{1.3 .(2 n-3)} \frac{2^{n-1}}{\pi} .
\end{aligned}
$$

The second moment $\mu_{2}(n)$ of $T G C(n, x)$ is

$$
\mu_{2}(n)-E\left(X^{2}\right)=\frac{1}{2 n-3}, n \geq 2 .
$$

The variance $\sigma^{2}(n)$ of $X$ is

$$
\sigma^{2}(n)=\frac{1}{2 n-3}-\left(\frac{(n-2) !}{1.3 .(2 n-3)} \frac{2^{n-1}}{\pi}\right)^{2}, n \geq 2 .
$$

The variance decreases as $n$ increases. The following recurrence relations hold for integer $m$, $1 \leq m<n, n \geq 4$

$$
\mu_{2 m}(n)=\frac{2 n-2}{2 n-3} \mu_{2 m-2}(n-1)-\mu_{2 m-2}(n)
$$

and

$$
\mu_{2 m+1}(n)=\frac{2 n-2}{2 n-3} \mu_{2 m-1}(n-1)-\mu_{2 m-1}(n) .
$$

Table 2 gives the percentile points of $\operatorname{TGC}(n, x)$ for $n=2,5$ and 10 .

\begin{tabular}{|c|c|c|c|}
\hline \hline$p$ & TGC(2) & TGC(5) & TGC(10) \\
0.1 & $7.8865 \times 10^{-2}$ & $4.3084 \times 10^{-2}$ & $2.9217 \times 10^{-2}$ \\
0.2 & 0.15974 & $8.6985 \times 10^{-2}$ & $5.8942 \times 10^{-2}$ \\
0.3 & 0.24491 & 0.13262 & $8.9748 \times 10^{-2}$ \\
0.4 & 0.33740 & 0.18116 & 0.12235 \\
0.5 & 0.44161 & 0.23424 & 0.15775 \\
0.6 & 0.56492 & 0.29447 & 0.19752 \\
0.7 & 0.72156 & 0.36657 & 0.24444 \\
0.8 & 0.94555 & 0.46101 & 0.3046 \\
0.9 & 1.3587 & 0.61104 & 0.39669 \\
\hline \hline
\end{tabular}

Journal home page: www.jafristat.net 
As $n$ increases the $p d f$ of $T G C$ concentrates towards origin. The hazard rate $h(n, x)$ of $T G C(n, x)$ is

$$
h(n, x)=\frac{1}{\left(1+x^{2}\right)^{n} B \frac{1}{1+x^{2}}\left(n-\frac{1}{2}, \frac{1}{2}\right)},
$$

where $\mathrm{B}_{x}(p . q)=\frac{\Gamma\left(\frac{1}{2}\right) \Gamma\left(n-\frac{1}{2}\right)}{\Gamma(n)} \int_{0}^{x} u^{p-1}(1-u)^{q-1} d x$.

Figure 3 gives $h(n, x)$ for $\mathrm{n}=2,5$ and $10, h(2, x)$-Red, $h(5, x)$-Green and $h(10, x)$-Black.

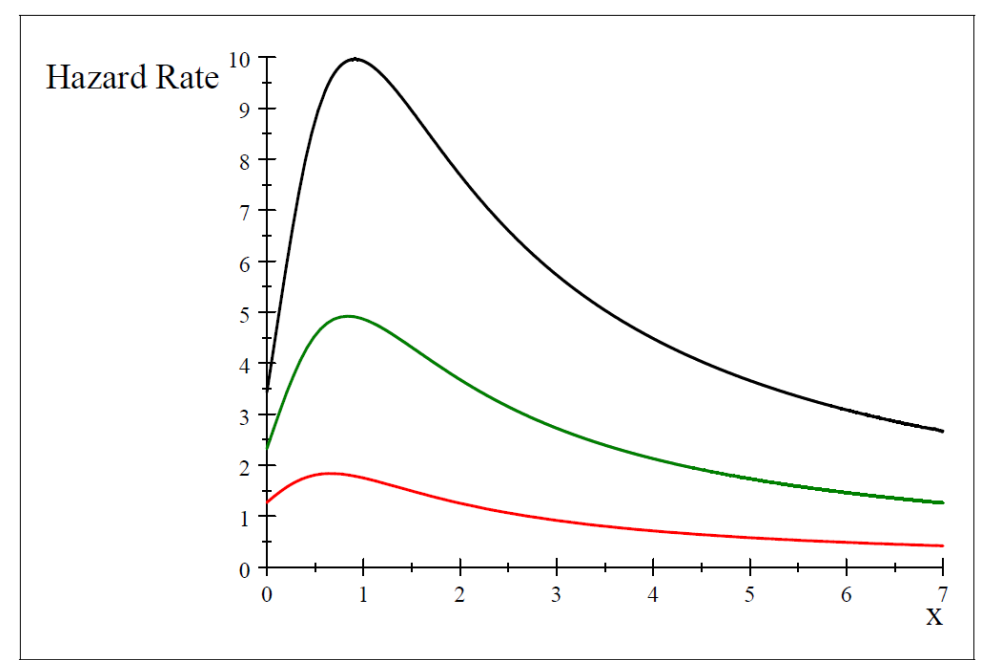

Fig. 3. Hazard Rate, $h(n, x)$

We will need the following two Lemmas to prove our characterization theorems.

Assumption $A$. Suppose the random variable $\mathrm{X}$ is absolutely continuous with cumulative distribution function (cdf) $\mathrm{F}(\mathrm{x})$ and probability density function (pdf) $f(x)$. We assume $\gamma=\{x \mid F(x)>0\}$ and $\delta=\inf \{x \mid F(x)<1\}$, We assume further that $E(X)$ exists.

Lemma 1. Under the assumption $A$ with $n \geq 2$. If

$$
E(X \mid X \leq x)=g(x) \tau(x)
$$

where $\tau(x)=\frac{f(x)}{F(x)}$ and $g(x)$ is a continuous differentiable function of $x$ with the condition

$$
\int_{0}^{x} \frac{u-g^{\prime}(u)}{g(u)} d u \text { is finite for all } x, \gamma<x<\delta
$$

then

$$
f(x)=c e^{\int_{0}^{x} \frac{u-g^{\prime}(u)}{g(u)} d u}, 0<x<\infty,
$$

where $c$ is determined by the condition $\int_{\gamma}^{\delta} f(x) d x=1$. 
Proof of Lemma 1. We have

$$
g(x)=\frac{\int_{0}^{x} u f(u) d u}{f(x)}
$$

i.e.

$$
\int_{0}^{x} u f(u) d u=f(x) g(x) .
$$

By differentiating with respect to respect to $x$, we obtain

$$
x f(x)=f^{\prime}(x) g(x)+f(x) g^{\prime}(x) .
$$

From the above equation, we obtain

$$
\frac{f^{\prime}(x)}{f(x)}=\frac{x-g^{\prime}(x)}{g(x)} .
$$

On integrating, we have

$$
f(x)=c e^{\int_{0}^{x} \frac{u-g^{\prime}(u)}{g(u)}} d u,
$$

where $c$ is obtained by the condition $\int_{\gamma}^{\delta} f(u) d u=1$.

Lemma 2. Under the assumption $A$, if

$$
E(X \mid X \geq x)=g(x) r(x)
$$

where $r(x)=\frac{f(x)}{1-F(x)}$ and $g(x)$ is a continuous differentiable function of $x$ with the condition

$$
\int_{x}^{\infty} \frac{u+g^{\prime}(u)}{g(u)} d u \text { is finite for all } x, \gamma<x<\delta
$$

then

$$
f(x)=c e^{-\int_{0}^{x} \frac{u+g^{\prime}(u)}{g(u)} d u}, 0<x<\infty,
$$

where $c$ is determined by the condition $\int_{\gamma}^{\delta} f(x) d x=1$.

Proof of Lemma 1. We have

$$
g(x)=\frac{\int_{x}^{\infty} u f(u) d u}{f(x)}
$$

i.e.

$$
\int_{x}^{\infty} u f(u) d u=f(x) g(x)
$$

Differentiating with respect to respect to $x$, we obtain

$$
-x f(x)=f^{\prime}(x) g(x)+f(x) g^{\prime}(x) .
$$

From the above equation, we obtain

$$
\frac{f^{\prime}(x)}{f(x)}=-\frac{x+g^{\prime}(x)}{g(x)} .
$$

On integrating, we have

$$
f(x)=c e^{-\int_{0}^{x} \frac{u x+g^{\prime}(u)}{g(u)}} d u,
$$

where $c$ is obtained by the condition $\int_{\gamma}^{\delta} f(u) d u=1$. 
Theorem 1. If the random variable $X$ satisfies the assumption $A$ with $\gamma=0$ and $\delta=\infty$, then

$$
E(X \mid X \leq x)=g(x) \tau(x)
$$

where $\tau(x)=\frac{f(x)}{F(x)}$ and $g(x)=\frac{1}{2(n-1)}\left(\left(1+x^{2}\right)^{n}-\left(1+x^{2}\right)\right)$, if and only if

$$
f_{t r c}(x)=\frac{2 \Gamma(n)}{\Gamma\left(\frac{1}{2}\right) \Gamma\left(n-\frac{1}{2}\right)} \frac{1}{\left(1+x^{2}\right)^{n}}, 0<x<\infty, n>1 .
$$

Proof of Theorem 1. If

$$
f_{t r c}(x)=\frac{2 \Gamma(n)}{\Gamma\left(\frac{1}{2}\right) \Gamma\left(n-\frac{1}{2}\right)} \frac{1}{\left(1+x^{2}\right)^{n}}, 0<x<\infty, n>1
$$

then

$$
\begin{aligned}
g(x) & =\frac{\int_{0}^{x} u f_{t r c}(u) d u}{f_{t r c}(x)}=\frac{\int_{0}^{x} \frac{u}{\left(1+u^{2}\right)^{n}} d u}{\frac{1}{\left(1+x^{2}\right)^{n}}} \\
& =\frac{1}{2(n-1)}\left(\left(1+x^{2}\right)^{n}-\left(1+x^{2}\right)\right)
\end{aligned}
$$

Suppose that

$$
g(x)=\frac{1}{2(n-1)}\left(\left(1+x^{2}\right)^{n}-\left(1+x^{2}\right)\right)
$$

then

$$
g^{\prime}(x)=\frac{x}{n-1}\left(n\left(x^{2}+1\right)^{n-1}-1\right)
$$

We have

$$
\begin{gathered}
x-g^{\prime}(x)=\frac{n x}{n-1}\left(1-\left(1+x^{2}\right)^{n-1}\right) \\
g(x)=\frac{1}{2(n-1)}\left(\left(1+x^{2}\right)^{n}-\left(1+x^{2}\right)\right. \\
\frac{x-g^{\prime}(x)}{g(x)}=\frac{-n x}{2\left(1+x^{2}\right)} .
\end{gathered}
$$

Thus by Lemma 1,

$$
\frac{f^{\prime}(x)}{f(x)}=\frac{-n x}{2\left(1+x^{2}\right)} .
$$

On integrating the above equation, we obtain

$$
f(x)=\frac{c}{\left(1+x^{2}\right)^{n}} .
$$

Using the conditions with $\int_{0}^{\infty} f(x)=1$, we obtain

$$
f(x)=\frac{2 \Gamma(n)}{\Gamma\left(\frac{1}{2}\right) \Gamma\left(n-\frac{1}{2}\right)} \frac{1}{\left(1+x^{2}\right)^{n}}, 0<x<\infty .
$$


Theorem 2. If the random variable $X$ statisfies the assumption $A$ with $\gamma=0$ and $\delta=\infty$. Then

$$
E(X \mid X \geq x)=g(x) r(x) .
$$

where $r(x)=\frac{f(x)}{1-F(x)}$ and $g(x)=\frac{1+x^{2}}{2(n-1)}$ if and only if

$$
f_{t r c}(x)=\frac{2 \Gamma(n)}{\Gamma\left(\frac{1}{2}\right) \Gamma\left(n-\frac{1}{2}\right)} \frac{1}{\left(1+x^{2}\right)^{n}}, 0<x<\infty, n>1
$$

\section{Proof of Theorem 2. If}

$$
f_{\text {trc }}(x)=\frac{2 \Gamma(n)}{\Gamma\left(\frac{1}{2}\right) \Gamma\left(n-\frac{1}{2}\right)} \frac{1}{\left(1+x^{2}\right)^{n}}, 0<x<\infty, n>1,
$$

then

$$
\begin{aligned}
g(x) & =\frac{\int_{x}^{\infty} u f_{\operatorname{trc}}(u) d u}{f_{\operatorname{trc}}(x)}=\frac{\int_{x}^{\infty} \frac{u}{\left(1+u^{2}\right)^{n}} d u}{\frac{1}{\left(1+x^{2}\right)^{n}}} \\
& =\frac{1+x^{2}}{2(n-1)} .
\end{aligned}
$$

Suppose that $g(x)=\frac{1+x^{2}}{2(n-1)}$, then $g^{\prime}(x)=\frac{x}{n-1}$ and we have

$$
x+g^{\prime}(x)=\frac{n x}{n-1}
$$

and

$$
\frac{x+g^{\prime}(x)}{g(x)}=\frac{2 n x}{1+x^{2}}
$$

Thus by Lemma 2,

$$
\frac{f^{\prime}(x)}{f(x)}=-\frac{2 n x}{1+x^{2}} .
$$

On integrating the above equation, we obtain

$$
\frac{f^{\prime}(x)}{f(x)}=\frac{2 n x}{\left(1+x^{2}\right)} \text {. }
$$

Using the conditions with $\int_{0}^{\infty} f(x)=1$, we obtain

$$
f(x)=\frac{2 \Gamma(n)}{\Gamma\left(\frac{1}{2}\right) \Gamma\left(n-\frac{1}{2}\right)} \frac{1}{\left(1+x^{2}\right)^{n}}, 0<x<\infty .
$$

Now, let $X_{1}, X_{2}, \ldots, X_{n}$ are $n$ independent copies of the random variable X having absolutely continuous distribution function $F(x)$ and $p d f f(x)$, and $X_{1, n} \leq X_{2, n} \leq,,, . \leq X_{n, n}$ are the corresponding order statistics. It is known (see Ahsanullah and Nevzorov, 2013, Chapter 1), and (Arnold et al., 2005, Chapter 1), that the $X_{j, n} \mid X_{k . n}=x$ for $1 \leq k<j \leq n$, is distributed as the $j-k-t h$ order statistics from $n-k$ independent observations from the random variable $Y$ having the $p d f \frac{f(u)}{1-F(x)}, 0 \leq u<x$. Further $X_{i . n} \mid X_{k, n}=x, 1 \leq i<k \leq n$ 
is distributed as $i-t h$ order statistics from $k$ independent observations from the random variable $W$ having the $p d f f_{W}(w \mid x)$ where

$$
f_{W}(w \mid x)=\frac{f(w)}{F(x)}, w<x .
$$

We assume

$$
S_{k-1}=\frac{1}{k-1}\left(X_{1, n}+X_{2, n}+\ldots+X_{k-1}\right)
$$

and

$$
T_{k, n}=\frac{1}{n-k}\left(X_{k+1, n}+X_{k+2, n}+\ldots+X_{n . n}\right) .
$$

We have the following :

Theorem 3. Suppose the random variable $X$ satisfies the assumption $A$ with $\gamma=0$ and $\delta=\infty$, then

$$
E\left(S_{k-1} \mid X_{k, n}=x\right)=g(x) \tau(x)
$$

where

and

$$
\tau(x)=\frac{f(x)}{F(x)}
$$

$$
g(x)=\frac{1}{2(n-1)}\left(\left(1+x^{2}\right)^{n}-\left(1+x^{2}\right)\right)
$$

if and only if

$$
f_{\text {trc }}(x)=\frac{2 \Gamma(n)}{\Gamma\left(\frac{1}{2}\right) \Gamma\left(n-\frac{1}{2}\right)} \frac{1}{\left(1+x^{2}\right)^{n}}, 0<x<\infty, n>1 .
$$

Proof of Theorem 3. If

$$
f_{t r c}(x)=\frac{2 \Gamma(n)}{\Gamma\left(\frac{1}{2}\right) \Gamma\left(n-\frac{1}{2}\right)} \frac{1}{\left(1+x^{2}\right)^{n}}, 0<x<\infty, n>1 .
$$

then

$$
E\left(S_{k-1} \mid X_{k, n}=x\right)=\frac{\int_{0}^{x} u f_{t r c}(u) d u}{F_{t r c}(x)}=\frac{\int_{0}^{x} \frac{u}{\left(1+u^{2}\right)^{n}} d u}{\frac{1}{\left(1+x^{2}\right)^{n}}} \tau(x)
$$

Thus

$$
g(x)=\frac{1}{2(n-1)}\left(\left(1+x^{2}\right)^{n}-\left(1+x^{2}\right)\right) .
$$

Suppose that

$$
E\left(S_{k-1} \mid X_{k, n}=x\right)=g(x) \tau(x) .
$$

with $g(x)=\frac{1}{2(n-1)}\left(\left(1+x^{2}\right)^{n}-\left(1+x^{2}\right)\right)$. But

$$
E\left(S_{k-1} \mid X_{k, n}=x\right)=\frac{\int_{0}^{\infty} u f(u) d u}{F(x)} .
$$

Thus

$$
g(x)=\frac{\int_{0}^{x} u f(u) d u}{f(u)}=\frac{1}{2(n-1)}\left(\left(1+x^{2}\right)^{n}-\left(1+x^{2}\right)\right)
$$

Journal home page: www.jafristat.net 
M. Ahsanullah, Afrika Statistika, Vol. 10(2), 2015, pages 815-826. Some inferences on truncated generalized Cauchy distribution.

Now

$$
g^{\prime}(x)=\frac{x}{n-1}\left(n\left(1+x^{2}\right)^{n-1}-1\right)
$$

and

$$
\frac{x-g^{\prime}(x)}{g(x)}=\frac{-n x}{2\left(1+x^{2}\right)} .
$$

Thus by Lemma 1,

$$
\frac{f^{\prime}(x)}{f(x)}=\frac{-n x}{2\left(1+x^{2}\right)} .
$$

On integrating the above equation, we obtain $f(x)=\frac{c}{\left(1+x^{2}\right)^{n}}$ and by using the condition $\int_{0}^{\infty} f(u) d u=1$, we obtain

$$
f(x)=\frac{2 \Gamma(n)}{\Gamma\left(\frac{1}{2}\right) \Gamma\left(n-\frac{1}{2}\right)} \frac{1}{\left(1+x^{2}\right)^{n}}, 0<x<\infty .
$$

Theorem 4. Suppose the random variable $X$ satisfies the assumption $A$ with $\gamma=0$ and $\delta=\infty$, then

$$
E\left(T_{k, n} \mid X_{k, n}=x\right)=g(x) r(x)
$$

where

$$
g(x)=\frac{1+x^{2}}{2(n-1)}
$$

and

$$
r(x)=\frac{f(x)}{1-F(x)}
$$

if and only if $X$ has the $p d f$

$$
f(x)=\frac{2 \Gamma(n)}{\Gamma\left(\frac{1}{2}\right) \Gamma\left(n-\frac{1}{2}\right)} \frac{1}{\left(1+x^{2}\right)^{n}}, 0<x<\infty .
$$

Proof of Theorem 4. Suppose that

$$
f(x)=\frac{2 \Gamma(n)}{\Gamma\left(\frac{1}{2}\right) \Gamma\left(n-\frac{1}{2}\right)} \frac{1}{\left(1+x^{2}\right)^{n}}, 0<x<\infty
$$

then we have

$$
\begin{aligned}
E\left(T_{k, n} \mid X_{k, n}\right. & =x)=\frac{\int_{x}^{\infty} u f(u) d u}{1-F(x)} \\
& =\frac{\int_{x}^{\infty} u f(u) d u}{f(x)} r(x) \\
& =\frac{\int_{x}^{\infty} \frac{u}{\left(1+u^{2}\right)^{n}} d u}{\frac{1}{\left(1+x^{2}\right)^{n}}} r(x) .
\end{aligned}
$$

Thus

$$
g(x)=\frac{\int_{x}^{\infty} \frac{u}{\left(1+u^{2}\right)^{n}} d u}{\frac{1}{\left(1+x^{2}\right)^{n}}}=\frac{1+x^{2}}{2(n-1)} .
$$

Journal home page: www.jafristat.net 
Suppose that $g(x)=\frac{1+x^{2}}{2(n-1)}$. We have

$$
\begin{aligned}
E\left(T_{k . n} \mid X_{k, n}\right. & =x)=\frac{\int_{x}^{\infty} u f(u) d u}{1-F(x)} \frac{\int_{x}^{\infty} u f(u) d u}{f(x)} r(x) \\
& =g(x) r(x) .
\end{aligned}
$$

Thus

$$
g(x)=\frac{\int_{x}^{\infty} u f(u) d u}{f(x)}=\frac{1+x^{2}}{2(n-1)} .
$$

We have

$$
g^{\prime}(x)=\frac{2 x}{2(n-1)}
$$

and

$$
\frac{x+g^{\prime}(x)}{g(x)}=\frac{2 n x}{1+x^{2}} .
$$

Using 2, we obtain

$$
f(x)=c e^{-\int \frac{2 n x}{1+x^{2}} d x} .
$$

Now, by using the condition $\int_{o}^{\infty} f(x) d x=1$, we obtain

$$
f(x)=\frac{2 \Gamma(n)}{\Gamma\left(\frac{1}{2}\right) \Gamma\left(n-\frac{1}{2}\right)} \frac{1}{\left(1+x^{2}\right)^{n}}, 0<x<\infty .
$$

Suppose that $X_{1}, X_{2}, \ldots$ is a sequence of independent and identically distributed absolutely continuous random variables with distribution function $F(x)$ and pdf $f(x)$. Let $Y_{n}=\max \left(X_{1}, X_{2}, \ldots, X_{n}\right)$ for $n \geq 1$. We say $X_{j}$ is an upper record value of $\left\{X_{n}, n \geq 1\right\}$ if $Y_{j}>Y_{j-1}$, for $j>1$. The indices at which the upper records occur are given by the record times :

$$
\left\{U(n)>\min \left\{j \mid j>U(n+1), X_{j}>X_{U(n-1)}, n>1\right\}\right.
$$

and $U(1)=1$. We will denote the $n t h$ upper record value $X(n)=X_{U(n)}$.

Theorem 5. Suppose the random variable $X$ satisfies the assumption $A$ with $\gamma=0$ and $\delta=\infty$. Then $X$ has the distribution $T G C(n, x)$ if and only if

$$
E(X(n+1) \mid X(n)=x)=g(x) r(x)
$$

where

$$
g(x)=\frac{1+x^{2}}{2(n-1)}
$$

and

$$
r(x)=\frac{f(x)}{1-F(x)} .
$$

Proof of Theorem 5. We have

$$
E(X(n+1) \mid X(n)=x)=\frac{\int_{x}^{\infty} u f(u) d u}{1-F(u)} .
$$

From there, the result follows from Theorem 2.

Journal home page: www.jafristat.net 
M. Ahsanullah, Afrika Statistika, Vol. 10(2), 2015, pages 815-826. Some inferences on truncated generalized Cauchy distribution.

\section{References}

Ahsanullah, M., 1995. Record Statistics. Nova Science Publishers. New-York,USA.

Ahsanullah, M. and Nevzorov, V.B., 2001. Ordered Random Variables. Nova Science Publishers, New York,USA.

Ahsanullah, M., Nevzorov, V.B. and Shakil,M., 2013. Order Statistics. Atlantis-Press, Paris,France.

Ateya, S.F. and Al-Hussaini, E.K., 2011. Inferences under truncated generalized Cauchy distribution. Journal of Applied Statistical Science, vol. 19(4), 357-374.

Arnold, B.C., Balakrishnan, and Nagaraja, H.N., 2005. First Course in Order Statistics. Wiley, New York,USA.

Rider, P.R., 1957. Generalized Cauchy distribution. Ann. Instit. Statist. Math. 9, 215-223. 